\title{
Electrodynamics of Electronic Paper Based on Total Internal Reflection
}

\author{
Bavo Robben, ${ }^{1, *}$ Filip Beunis, ${ }^{1}$ Kristiaan Neyts, ${ }^{1}$ Robert Fleming, ${ }^{2}$ Bram Sadlik, ${ }^{3}$ Thomas Johansson, ${ }^{3}$ \\ Lorne Whitehead, ${ }^{4}$ and Filip Strubbe ${ }^{1}$ \\ ${ }^{1}$ Electronics and Information Systems Department and Center for Nano and Biophotonics, Ghent University, \\ Technologiepark Zwijnaarde 15, 9052 Zwijnaarde, Belgium \\ ${ }^{2}$ CLEARink Displays, 4020 Clipper Court, Fremont, California 94538, USA \\ ${ }^{3}$ CLEARink Displays, 123 Cambie Street, Suite 600, Vancouver, British Columbia, Canada V6B 1 B8 \\ ${ }^{4}$ University of British Columbia, 6224 Agricultural Road, Vancouver, British Columbia, Canada V6T 1Z1
}

(Received 21 March 2018; revised manuscript received 9 August 2018; published 20 September 2018)

\begin{abstract}
We present a study of the fundamental electrodynamics of an electronic ink pixel based on frustrated total internal reflection, which is relevant for the ongoing development of video-speed-capable electronic ink displays. Devices are fabricated with plane-parallel electrodes covered with a dielectric coating of the poly(p-xylylene) polymer Parylene $\mathrm{C}$ and filled with ink proprietary fluorochemical fluid containing absorbing particles. Reflectivity measurements of frustrated total internal reflection and transient current measurements provide information on the concentration of particles near the electrodes, conductivity of the ink, and screening of the electric field. By comparing the measurements with an electro-optical model, it is found that the electrical properties of the ink are dominated by positively charged particles and negative countercharges. Screening of the electric field depends as expected on the dielectric coating thickness. The observation of an asymmetry in the steady-state gray level response to an applied voltage is explained by fixed positive charges adsorbed at the interface between the dielectric coating and the dispersion. The overall switching dynamics of the device for different voltage sequences is in good agreement with simulations.
\end{abstract}

DOI: 10.1103/PhysRevApplied.10.034041

\section{INTRODUCTION}

In recent years there has been a rapid development in smart portable outdoor-readable devices [1]. Current display technologies used in these devices suffer from relative high power consumption and low contrast outdoors. This has created a demand for full-color video-speed reflective displays. Introducing video speed to electrophoretic displays and electrowetting displays is currently under investigation $[1,2]$.

Most commercially available electrophoretic ink displays are based on the electrophoretic displacement of oppositely charged absorbing (black) and scattering (white) particles over distances of the order of tens or hundreds of micrometers [3]. The resulting switching frequency is lower than $10 \mathrm{~Hz}$ and may be acceptable for reading, for signage, and other applications with low information content, but is too slow to play videos [1]. Electronic ink displays based on total internal reflection (TIR) may be the key for realizing fast, video-speed electronic paper. By adopting the principle of TIR, a highly reflecting white state can be achieved in the absence of absorbing particles in the evanescent field, while a dark state

*bavo.robben@ugent.be is obtained when absorbing particles are present in the evanescent field (see Fig. 1). Considering that the evanescent field penetrates only a few hundred nanometers into the liquid, the distance over which particles have to be transported is more than 100 times smaller than in conventional electrophoretic displays. As a result, the motion of two kinds of pigment particles across the whole pixel thickness in conventional electrophoretic ink displays can be replaced by moving one type of particle over only hundreds of nanometers near the surface, allowing much faster switching [4]. In general, such a TIR-based electronic paper uses absorbing pigment particles in a low refractive index low dielectric strength liquid, and incorporates a structured (hemispheres or pyramids) high to low refractive index substrate-liquid interface to enhance the fraction of ambient light experiencing TIR [5]. By using a structured glass-liquid interface, incident light can be reflected at multiple interfaces before reaching the observer and the contrast ratio between black and white can be increased.

Switching of electronic ink displays, either conventional or TIR-based, is based on the field-induced motion of charged colloidal particles in a nonpolar liquid. Many physical mechanisms involved in the switching of electronic ink displays have been investigated over the last decade [6,7]. The electronic inks usually contain 


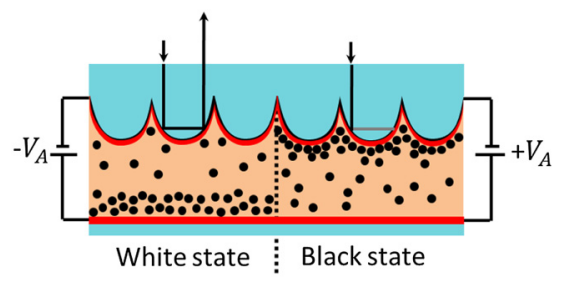

FIG. 1. Working principle of an electronic ink display based on TIR. The liquid (orange) has a lower refractive index than the glass (blue). The two substrates are covered with an electrode (black) and dielectric coating (red). When a negative voltage is applied (white state, left side) there are few particles near the top substrate and TIR occurs twice. When a positive voltage is applied (black state, right side) particles are present in the evanescent field and absorb the incident light.

surfactants and other additives to electrically charge and stabilize pigment particles. As a side effect of these surfactants, other charged species, such as charged inverse micelles, are introduced which affect the switching dynamics $[8,9]$. On the topic of charged inverse micelles, many studies have focused on properties such as concentration and electrophoretic mobility [10], generation and recombination [11], interactions with interfaces [12], the influence of dielectric coatings [13], and steady-state and dynamic switching regimes [14]. On the topic of charged colloidal particles in nonpolar liquids, there are many investigations of the charging mechanisms mediated by charged or uncharged inverse micelles [15-17] and switching of particles $[18,19]$. It should be noted that many of these studies are performed by measuring individual tracer particles. Even though there are some accounts of the switching of actual electronic inks [20], these are mostly experimental in nature, lacking a more fundamental understanding.

In this paper, we study the electro-optical switching of an electronic ink pixel based on TIR, with an ink having a high particle concentration resulting in a high contrast ratio. Through experiments and simulations the aim is to achieve a better understanding of the electrical and optical mechanisms involved in switching. These insights are valuable for improving contrast, switching speed, and consistency of gray levels of TIR-based electronic ink displays and of conventional electrophoretic ink displays. The motion of the pigment particles and other charged species in response to a sequence of voltage pulses is investigated by measuring the electrical current as well as the optical reflectivity as a function of time. The experimental results are compared with the results of a numerical electro-optical simulation program. The analysis shows that the motion of charged inverse micelles and charged pigment particles in the bulk can be accurately described by drift and diffusion. The results suggest that the liquid only contains positively charged pigment particles and negative countercharges. We demonstrate that a dielectric coating covering the electrodes leads to screening of the electric field and that this mechanism allows to sustain gray levels with low power consumption. Finally, we show that some charged species may be adsorbed at the solid-liquid interfaces, which has a large influence on the switching dynamics and the gray levels.

In display applications, electrophoretic devices based on TIR with a wide viewing angle can be achieved with hemispherical surface structures [5]. In this work, in order to facilitate the analysis, we investigate a planar configuration and use a prism to couple a laser beam into (and out of) the substrate at a high angle of incidence.

\section{MATERIALS AND METHODS}

The ink proprietary fluorochemical fluid used in this study (provided by Merck KGaA) contains absorbing particles of $195-\mathrm{nm}$ diameter. The refractive index of the mixture before adding the particles is about 1.3. The colloidal dispersion is inserted in a device consisting of two parallel, indium-tin-oxide (ITO)-coated glass $(n=1.51)$ substrates, separated by spherical glass spacers with a diameter $d$ of $25 \mu \mathrm{m}$. The ITO electrodes are covered with a vacuum-deposited dielectric coating of the poly(pxylylene) polymer Parylene $\mathrm{C}$ with a thickness $d_{s} / 2$ of either 50 or $200 \mathrm{~nm}$. The overlapping area of the electrodes $S$ is about $6.25 \mathrm{~cm}^{2}$. The device is sealed with UV curing glue (Norland, NOA 68) to prevent evaporation during measurements. Transient current measurements are performed with a Keithley 428 current amplifier while driving the cell with polarizing voltages (switch on to $V_{A}$ ) and voltage steps (switch from $V_{P}$ to $V_{A}$ ). The device is shielded from external electrical interference in order to obtain picoampere resolution. TIR measurements are carried out to investigate the particle density near the reflecting solid-liquid interface. TIR is achieved by coupling in an expanded laser beam (continuous, CrystaLaser $532 \mathrm{~nm}$ ) via a $45^{\circ}$ glass prism which is in optical contact

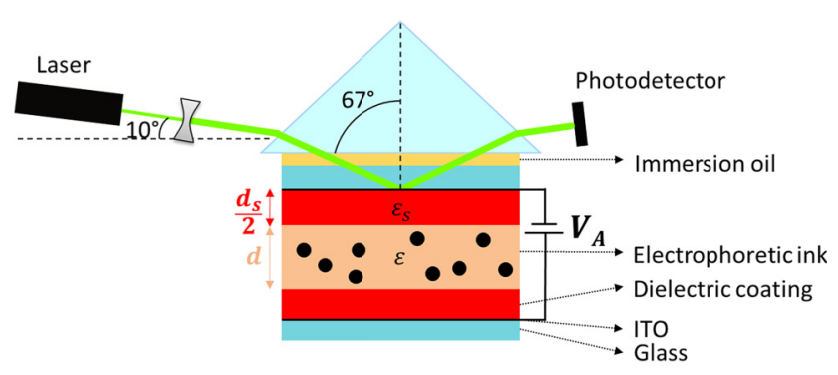

FIG. 2. Setup for measuring the reflectivity of an electrophoretic device under TIR, when a potential difference $V_{A}$ is applied. The incident laser beam $(532 \mathrm{~nm})$ makes an angle of $10^{\circ}$ with the horizontal and is attenuated and expanded to $5 \mathrm{~mW}$ with a $1-\mathrm{mm}$ diameter. A $45^{\circ}$ prism is in optical contact with the top glass substrate by immersion oil, allowing incidence at $67^{\circ}$, above the critical angle of $59^{\circ}$ needed for TIR. The intensity of the reflected beam is measured with a photodetector. 
with the top glass substrate using immersion oil (Nikon, type A) as illustrated in Fig. 2. The laser power is reduced to less than $5 \mathrm{~mW}$ by a neutral density filter. The angle of incidence in air is $10^{\circ}$ from the horizontal $\left(35^{\circ}\right.$ from the prism normal), while the angle of incidence on the glassliquid interface is $67^{\circ}$ from the normal, which is above the critical angle of $59^{\circ}$ for TIR. At the detector, the reflected laser beam has a diameter on the order of one square millimeter, which overfills the photodiode area (Thorlabs, DET10A/M) in order to allow reproducible measurements. Calibration of the data is done by normalization over the maximum reflectivity acquired in each dataset. Data acquisition for both electrical and reflectivity measurements is achieved with a NI DAQ device.

\section{MEASUREMENTS}

\section{A. Transient current measurements}

Transient current measurements provide valuable information on the transport of charges between the electrodes. Current measurements are carried out on devices that have a dielectric coating with a thickness of either 50 or $200 \mathrm{~nm}$ on both ITO electrodes. Prior to each measurement, the electrodes are short circuited for $500 \mathrm{~s}$ to ensure that all particles have returned to the equilibrium situation. Then, at $t=0$, the voltage applied across the electrodes is switched from $0 \mathrm{~V}$ to $V_{A}=0.1,0.2,0.5,1$, or $2 \mathrm{~V}$. The resulting transient currents for the coating thicknesses of 50 and $200 \mathrm{~nm}$ are shown in Fig. 3(a). Between 0 and $50 \mathrm{~ms}$, the currents are exponentially decreasing, with a time constant of 47 and $11 \mathrm{~ms}$, respectively, for coating thicknesses of 50 and $200 \mathrm{~nm}$. Figure 3(b) illustrates that the time constants $\tau$ are independent of the applied voltage. When we neglect the thickness of the insulator, we can estimate the conductivity of the mixture in equilibrium from the initial current using $\sigma=I(t=0 \mathrm{~s}) d / S V_{A}$. As expected, the bulk conductivity is independent of the applied voltage and the coating thickness with a value of $135 \mathrm{nS} / \mathrm{m}$ [see Fig. 3(c)].

\section{B. Reflectivity measurements}

Reflectivity measurements based on TIR provide information on the particle density in the evanescent field region extending a few hundred nanometers into the liquid medium. Information is obtained from only one of the two solid-liquid interfaces, but by applying voltage pulses with the opposite polarity, complementary information can be obtained. Typical measurements are based on voltage sequences between -10 and $10 \mathrm{~V}$. The duration of each applied voltage is $10 \mathrm{~s}$. It is verified that $10 \mathrm{~s}$ per step is sufficient to achieve quasi-equilibrium in reflectivity, giving approximately the same results as with $100 \mathrm{~s}$ per step. It is verified as well that no bistability is present, although the reflectivity variation depends on both the applied voltage $V_{A}$ and the preceding quasi-equilibrium state at voltage $V_{P}$. On the other hand, the quasi-equilibrium state only depends on the applied voltage $V_{A}$. All measurements are carried out on the devices with 200- and 50-nm dielectric coatings. Reflectivity values are normalized to the one for the brightest white measured in a complete dataset.

Figure 4 shows a series of reflectivity measurements for a device with 200-nm dielectric coatings on the electrodes. When switching from $V_{P}=10 \mathrm{~V}$ (dark state, reflectivity about 0.05 ) to voltages $V_{A}$ between $10 \mathrm{~V}$ and $-10 \mathrm{~V}$, a transition is observed toward a brighter steady state [Fig. 4(a)]. The transition takes about $50 \mathrm{~ms}$ for small voltage steps $\Delta V=V_{A}-V_{P}$ (for example, $\Delta V=2 \mathrm{~V}$ ). For larger voltage steps, the transition becomes faster (e.g.,
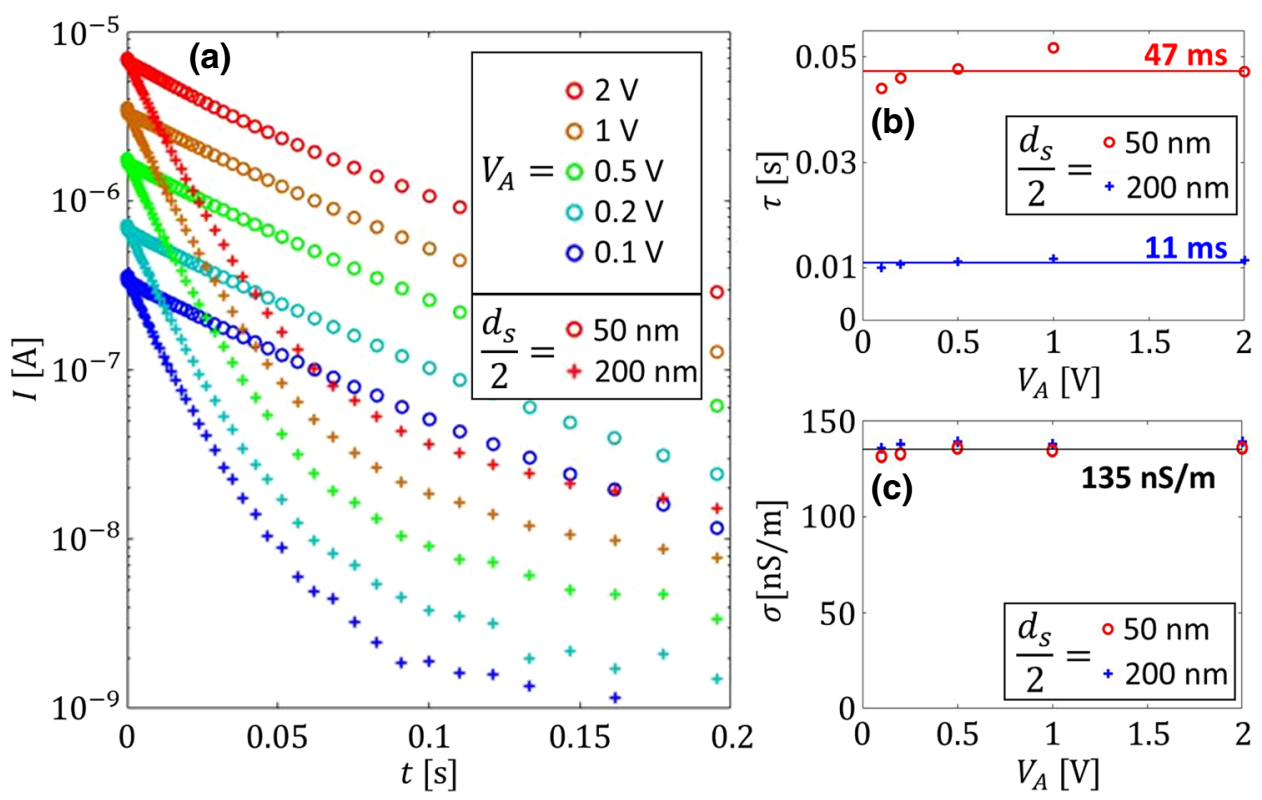

FIG. 3. (a) Current measurements after the voltage is switched on $\left(0 \mathrm{~V}\right.$ to $\left.V_{A}\right)$ at $t=0 \mathrm{~s}$ for devices with a 50- (o) or 200$\mathrm{nm}(+)$ dielectric coating on the electrodes. (b) Time constants $\tau$ obtained by exponential fitting between 0 and $50 \mathrm{~ms}$ in (a). (c) Estimated equilibrium conductivity $\sigma$ obtained from the initial current at $t=0$ in (a). 
(a)
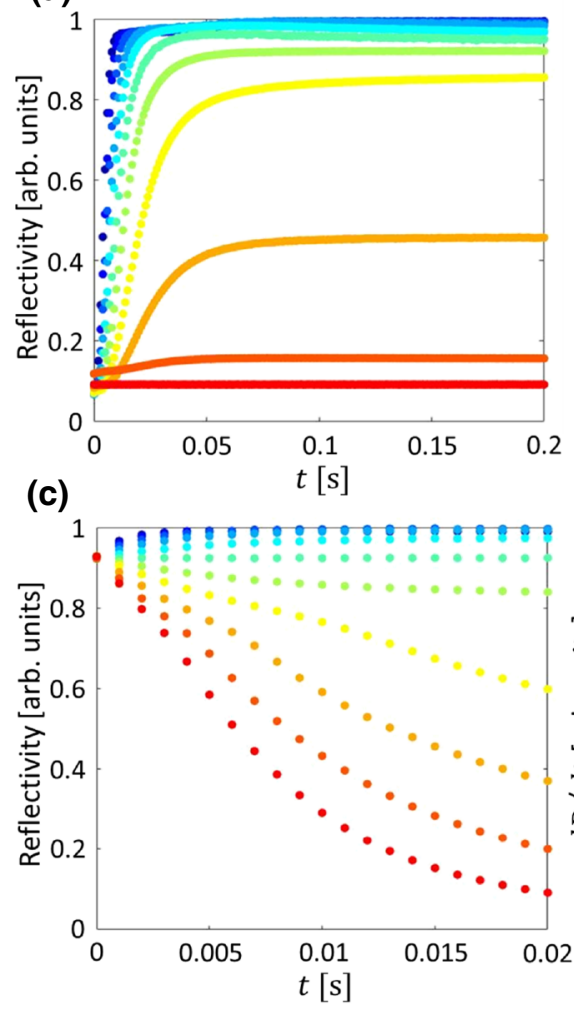

(d)
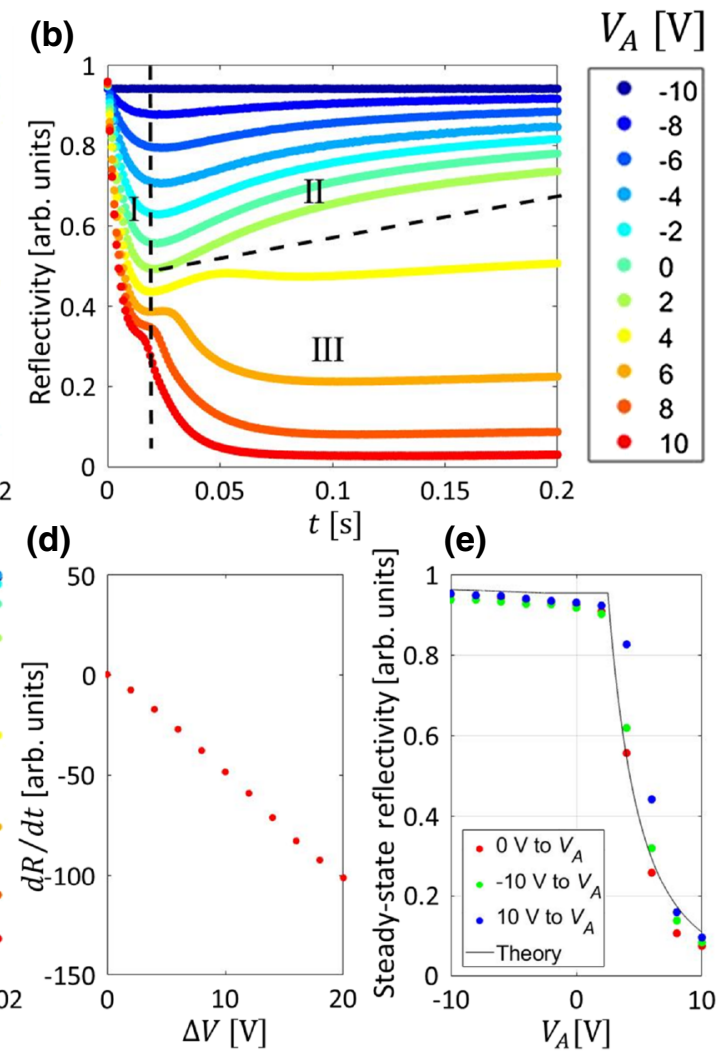

FIG. 4. Reflectivity measurements for a device with 200-nm dielectric coatings; (a) switching from $10 \mathrm{~V}$ to $V_{A}$, a fast black to white/gray transition; (b) switching from $-10 \mathrm{~V}$ to $V_{A}$ revealing three regions: region I initial decreasing reflectivity depending on $\Delta V$; region II increasing reflectivity toward white steady-state; region III decreasing reflectivity toward gray steady-state. In (c), the switch from $0 \mathrm{~V}$ to $V_{A}$ is shown for a short time scale. (d) The time derivative of the reflectivity $(d R / d t)$ at $t=0$ when switching from $-10 \mathrm{~V}$ to $V_{A}$ [from region I of (b)] is shown as a function of the voltage step $\Delta V=$ $V_{A}-V_{P}$. In (e) steady-state measurements after $10 \mathrm{~s}$ are given together with a theoretical model (line) showing a threshold at $2.5 \mathrm{~V}$. All reflectivities are normalized to the highest reflectivity measured in this dataset.
$10 \mathrm{~ms}$ for $\Delta V=20 \mathrm{~V})$. In Fig. 4(b), the device is switched from the steady-state situation at $V_{A}=-10 \mathrm{~V}$ to various voltages between -10 and $10 \mathrm{~V}$. The initial slope in the reflectivity vs time before $t=20 \mathrm{~ms}$ (region I) depends on the magnitude of the voltage step $\Delta V$ as illustrated in Fig. 4(d). In region II, the reflectivity increases again, leading to a minimum around $t=20 \mathrm{~ms}$, while in region III, the reflectivity further decreases. Switching from equilibrium at $V_{P}=0 \mathrm{~V}$ to various positive and negative voltages is shown in Fig. 4(c). The equilibrium situation at $V_{A}=$ $0 \mathrm{~V}$ is a bright state with 0.93 reflectivity. By applying a negative voltage the reflectivity increases. The maximum of reflectivity is reached for $V_{A}=-10 \mathrm{~V}$, which is used as a reference ( reflectivity of 1). Figure 4(e) shows the steady-state reflectivity obtained after $10 \mathrm{~s}$ as a function of the applied voltage. For $V_{A} \leq 0 \mathrm{~V}$, the reflectivity is about 0.93 . Note that the highest reflectivity of 1 shown in Fig. 4(c) has decreased toward 0.93 in the steady state. For $V_{A}>0 \mathrm{~V}$, there is a threshold of about $2.5 \mathrm{~V}$ above which the reflectivity decreases to a lower value. Since the reflectivity is measured at the grounded front side while the voltage $V_{A}$ is applied at the back, a low reflectivity for positive voltages indicates that the pigment particles are positively charged.

These measurements demonstrate the fast switching capability of a TIR-based electrophoretic display. Figure 4 shows that, depending on the applied voltages, switching times between different gray levels below $50 \mathrm{~ms}$ can be achieved. By optimization of the ink properties and device geometry, the switching time can be further reduced. At the same time, this measurement technique can be used to study micelle behavior and particle compaction at the surface for the case of conventional electrophoretic ink displays.

\section{ELECTRO-OPTICAL MODEL}

In order to explain the observed behavior, an electrooptical model is proposed. Electrically, the region between the electrodes is modeled by two ideal dielectric layers of thickness $d_{s} / 2$ and dielectric constant $\varepsilon_{s}$ (see Fig. 5) and a liquid layer containing charges with thickness $d$ and dielectric constant $\varepsilon$. At the dielectric-liquid interfaces, fixed surface concentrations $N_{l+}$ (left) $=N_{r+}$ (right) $=N_{+}$ of positive charges are present. The ink behaves as an electrophoretic medium with positively charged pigment particles and (small) negative countercharges. No other positive charges than these pigment particles are taken into account in the liquid. This assumption is evaluated in the discussion section.

The pigment particles are modeled as spheres with hydrodynamic radius $r$, concentration $n_{P}(x)$, electrophoretic mobility $\mu_{p}$, diffusion coefficient $D_{p}$, and charge per particle $Z_{p} e$. The countercharges are monovalent and have a concentration $n_{-}(x)$, mobility $\mu_{-}$, and 


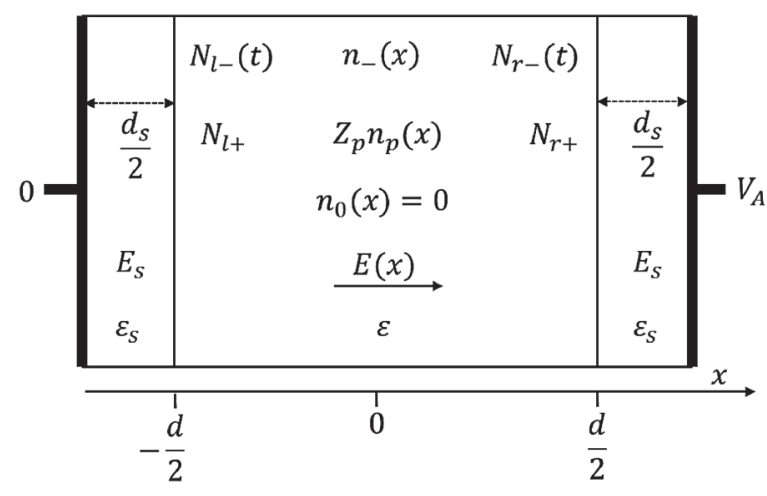

FIG. 5. Device structure showing the parameters used in the electro-optical model: bulk densities $n$, surface densities $N$, thicknesses $d$, fields $E$, dielectric constants $\varepsilon$, and voltage $V_{A}$.

diffusion coefficient $D_{-}$. The effect of gravity on the particles can be neglected.

Drift and diffusion in the bulk is governed by the Nernst-Planck equation, leading to a flux $\Psi$ for the countercharges:

$$
\Psi_{-}=-\mu_{-} n_{-} E-D_{-} \frac{\partial n_{-}}{\partial x} .
$$

For the pigment particles, an adapted Nernst-Planck equation is used to cope with steric hindrance:

$$
\Psi_{p}=f_{\mathrm{st}} \mu_{p} n_{p} E-D_{p} \frac{\partial n_{p}}{\partial x}
$$

where $f_{\text {st }}$ is the correction factor to cope with the steric hindrance. When an electric field accumulates pigment particles at the interface, a diffuse double layer is formed because of the equilibrium between drift and diffusion. However, there is a maximum allowed particle concentration $n_{\max }$ due to steric hindrance and it is determined by the closest sphere packing. This condition is implemented analytically in Eq. (2) by reducing the drift by a factor $f_{\text {st }}=\left[1-\left(n_{p} / n_{\max }\right)\right]$ when the particle concentration $n_{p}$ is approaching $n_{\max }$. When the particle concentration approaches the maximum value, the influx becomes zero and further compaction is prohibited. In literature, steric corrections for ionic transport and micelles are implemented by an additional diffusion term [21]. For the larger particles we investigate here, the diffusion is smaller and this approach may lead to numerical instabilities. In our case, the use of a reduced drift term results in a stable implementation. Due to the small particle concentration in the bulk region, the correction factor $\left[1-\left(n_{p} / n_{\max }\right)\right]$ remains close to unity. The transition region between the (low) bulk concentration and the maximum concentration is typically less than $40 \mathrm{~nm}$ wide. Therefore, we expect that this model will be a good approximation for the actual behavior.
The time evolutions of the concentrations of particles and countercharges in the bulk are determined, respectively, by the continuity equations $\partial n_{p} / \partial t=-\left(\partial \Psi_{p} / \partial x\right)$ and $\partial n_{-} / \partial t=-\left(\partial \Psi_{-} / \partial x\right)$. The electric field is governed by the Poisson equation:

$$
-\frac{\partial E}{\partial x}=\frac{\partial^{2} V}{\partial x^{2}}=-\frac{\rho}{\varepsilon \varepsilon_{0}},
$$

where $\rho=e Z_{p} n_{p}(x)-e n_{-}(x)$ and $V$ is the electric potential.

The solid-liquid interface is modeled as a blocking boundary for pigment particles by setting the particle flux $\left(\Psi_{p}\right)$ zero at the interfaces:

$$
\Psi_{p}\left(x=-\frac{d}{2}\right)=0 ; \Psi_{p}\left(x=\frac{d}{2}\right)=0 .
$$

If an electric field is present at the solid-liquid interfaces, different kinds of interaction between the charges and the solid surface can occur. Two boundary conditions often observed in inverse micelle studies are considered here: adsorption/release and a blocking boundary [12,22]. It can be mentioned here that both boundary conditions lead to similar results. For the adsorption/release boundary condition, adsorbed negative countercharges lead to surface concentrations $N_{l-}$ and $N_{r-}$, respectively, at the left and right solid-liquid interfaces. Next, we focus on the left interface, but similar equations apply to the right interface. Adsorption of negative countercharges having concentration $n_{l}$ near the left solid-liquid interface occurs if the electric field is positive:

$$
\frac{d N_{l-}}{d t}=\mu_{-} n_{l} E_{l}+D_{-} \frac{\partial n_{l}}{\partial x}, E_{l}<0 .
$$

The flux related to adsorption can be calculated from $-\Psi_{-}[x=-(d / 2)]=\left(d N_{l-} / d t\right)$.

Release of negative countercharges at the left solidliquid interface occurs as long as there is charge available on the surface $\left(N_{l-}>0\right)$. The released charge is determined such that the field is zero at the interface $[12,23]$ :

$$
\begin{aligned}
& N_{l-}= N_{l+}+\frac{\varepsilon_{0} \varepsilon_{s}}{d_{s} e} \\
& \times {\left[V_{A}+\int_{-(d / 2)}^{d / 2} \frac{Z_{p} n_{p} e-n_{-} e}{\varepsilon_{0} \varepsilon}\left(\frac{d}{2}-x\right) d x\right] } \\
& E_{l}=0 \\
& N_{l-}>0
\end{aligned}
$$

Only if the reservoir of adsorbed charges is empty $\left(N_{l-}=\right.$ $0)$ can the field become negative at the left interface:

$$
N_{l-}=0, \quad E_{l}<0 .
$$

For the other case of a blocking boundary condition, the same boundary condition is used as for the particles in 
Eq. (4), corresponding to the surface being a physical barrier keeping the charge flux zero.

The total charge should vanish due to neutrality:

$$
\int_{-(d / 2)}^{d / 2}\left(Z_{p} n_{p}-n_{-}\right) d x-N_{l-}-N_{r-}+N_{l+}+N_{r+}=0 .
$$

The optical response as obtained by TIR can be analyzed based on the internal particle distribution. In a simplified optical model, particles in the evanescent zone absorb light proportionally to their concentration and to the intensity of the local electromagnetic radiation. For simplicity we neglect the fact that the particles have a different refractive index and that the intensity is modified by the absorbing particles. We then approximate the reflectivity $R$ by an overlap integral of the exponentially decaying evanescent field with depth $\delta_{\mathrm{ev}}$ of the order of $100 \mathrm{~nm}$ and the normalized particle concentration $p(x)=\left(n_{p}(x) / n_{\max }\right)$, assuming that a maximally packed layer perfectly absorbs the evanescent field:

$$
R=1-\int \frac{1}{\delta_{\mathrm{ev}}} e^{-\left(x / \delta_{\mathrm{ev}}\right)} p(x) d x .
$$

\section{NUMERICAL SIMULATIONS}

The system of partial differential equations is solved for the appropriate interval for $x$ for increasing time $t$. Equations. (1)-(3) are solved for the bulk region using a first order discretized drift and diffusion model for positive particles and negative inverse micelles [24].

The motion of the particles is described by the density distribution $n_{p}$. To cope with a maximal concentration restriction due to a close packing limit, a reduction of particle flux is implemented [see Eq. (2)]. For the random close packing of monodisperse spheres [25], a volume percent of $64 \%$ is used in simulations. The boundary condition for pigment particles is a blocking condition, meaning that the total flux by drift and diffusion toward the surface is zero, without adsorption [Eq. (4)]. As a boundary condition for the countercharges, both blocking and an adsorption/release boundary condition have been examined, and both give satisfying results.
Physical parameters for the simulation of the ink are the particle diameter of $195 \mathrm{~nm}$, the effective particle charge of $Z_{p}=60$ (charge $60 e$ per particle), an initial particle concentration $n_{p}$ of $7.7 \times 10^{18} / \mathrm{m}^{3}$, the diameter of the countercharges (inverse micelles) is $7.2 \mathrm{~nm}$, the viscosity of the solvent is $4.5 \mathrm{mPa} \mathrm{s}$, and the relative dielectric constant of the solvent is 2 . Other relevant parameters are the cell thickness $(d=25 \mu \mathrm{m})$, dielectric coating thickness $\left(d_{s} / 2=200 \mathrm{~nm}\right)$, and dielectric constant of the coating $\left(\varepsilon_{s}=3\right)$. One additional parameter is the fixed charge on the surfaces of $N_{+} e=166 \mu \mathrm{C} \mathrm{m}^{-2}$. For the TIR we use $\delta_{\mathrm{ev}}=100 \mathrm{~nm}$.

Simulation results for the 200-nm dielectric coating with adsorption/release boundary conditions for negative charges when switching from $10 \mathrm{~V}$ to $V_{A}$ are shown in Figs. 6 and 7. The time-dependent reflectivity of both sides of the structure is given as well as the initial field $\left(t=10^{-6} \mathrm{~s}\right)$, the field at $t=0.02 \mathrm{~s}$, and at $t=0.2 \mathrm{~s}$.

\section{ANALYSIS OF THE SIMULATION RESULTS}

The simulations in Fig. 6 show a similar behavior as that observed in the measurements of Figs. 4(a) and 4(b). We present here the physical background of the regimes based on the model and simulation. If possible, we present simplified analytical solutions for the behavior.

\section{A. Analytical model for steady-state gray levels}

From the above equations, an analytical model can be constructed to examine the electro-optical state in equilibrium at an applied voltage $V_{A}$.

When there is no potential difference $\left(V_{A}=0 \mathrm{~V}\right)$, negative charges from the bulk are attracted by the fixed positive surface charge, accumulate at both interfaces until the positive surface charge is fully compensated $\left(N_{r-}=\right.$ $N_{l-}=N_{r+}=N_{l+}$ ), and the pigment particles and the remaining negative charges are homogeneously distributed in the bulk.

When a small positive voltage is applied, negative surface charges are released from the left side leaving behind a positively charged interface, while at the right interface, negative charges from the bulk are adsorbed. This process stops when a charge $Q=\varepsilon_{0} \varepsilon_{S} V_{A} S / d_{S}$ has been transferred (a)

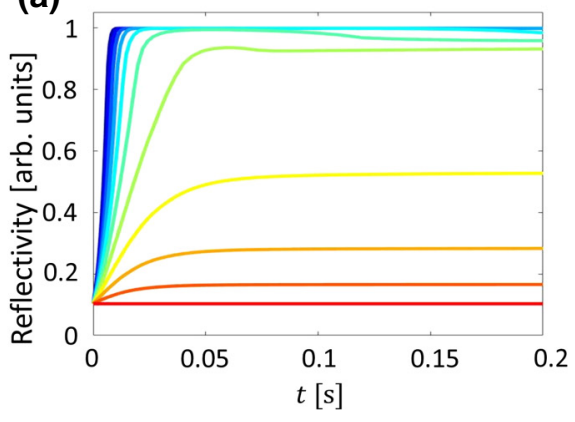

(b)

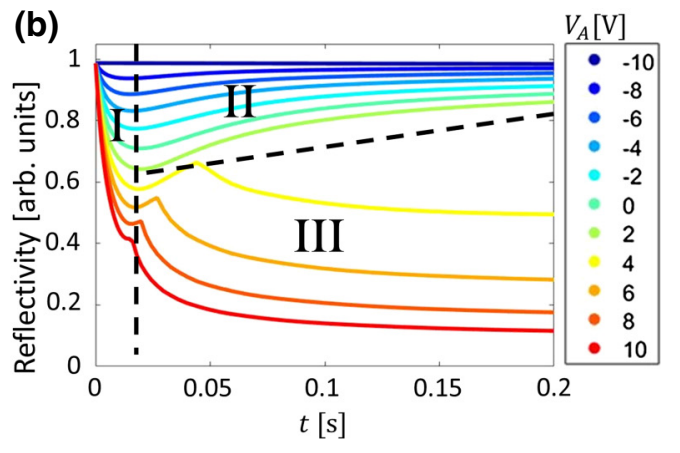

FIG. 6. Simulated reflectivity for a cell with a 200 -nm dielectric coating and adsorption/release boundary conditions, (a) switching from $10 \mathrm{~V}$ to $V_{A}$ in steps of $2 \mathrm{~V}$; (b) switching from $-10 \mathrm{~V}$ to $V_{A}$, three regions are indicated. 

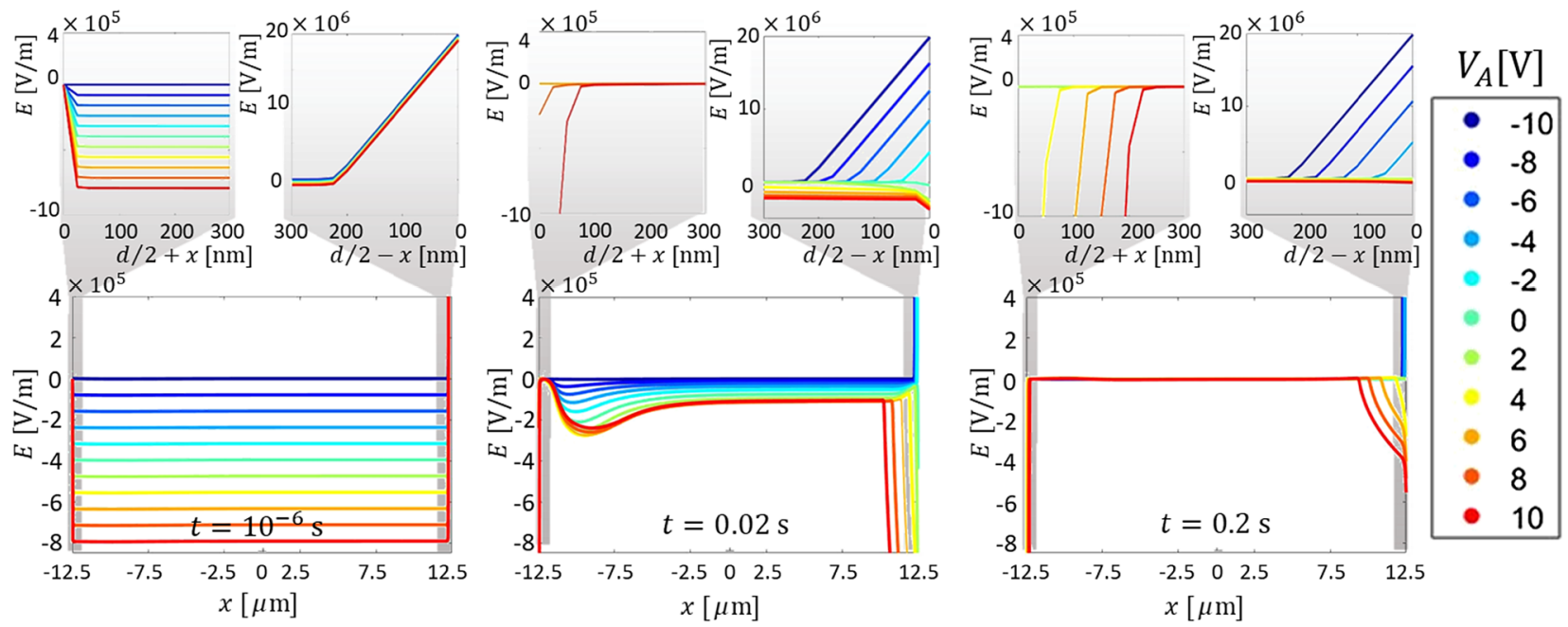

FIG. 7. Simulated electric field $E(x)$ of a cell with a $200-\mathrm{nm}$ dielectric coating and adsorption/release boundary condition, switching from $-10 \mathrm{~V}$ to $V_{A}$ in steps of $2 \mathrm{~V}$, at $t=10^{-6} \mathrm{~s}$ (left), $t=0.02 \mathrm{~s}$ (middle), and $t=0.2 \mathrm{~s}$ right. Bulk electric fields are shown below, surface fields above.

from the left to the right interface, because then the complete applied voltage drops across the two dielectric layers and the bulk field becomes zero again.

For a sufficiently large positive voltage, all negative charges are released. This occurs at the threshold voltage $V_{\text {thresh}}$.

$$
V_{\text {thresh }}=\frac{N_{+} e}{\varepsilon_{0} \varepsilon_{s}} d_{s}
$$

When the voltage is increased above $V_{\text {thresh }}$, we obtain in good approximation a layer of compacted particles with thickness $P$ and homogeneous charge density at the left interface as shown in Fig. 8. The variation in the electric field in Fig. 8 is similar to the result of the numerical simulation in Fig. 7. In steady state, the thickness $P$ is related to the applied voltage by

$$
E_{s} d_{s}+\frac{Z_{p} e n_{\max } P^{2}}{2 \varepsilon_{0} \varepsilon}=-V_{A},
$$

where $E_{S}$ is the electric field in the insulating layers given by

$$
E_{s}=-\frac{Z_{p} e n_{\max } P}{\varepsilon_{0} \varepsilon_{s}}-\frac{N_{+} e}{\varepsilon_{0} \varepsilon_{s}} .
$$

In this analytic model, the particle concentration is $n_{\max }$ (compacted) in the region with thickness $P$, and equal to $p_{0}$ elsewhere (noncompacted), with $p_{0}$ depending on the initial concentration $n_{p}$ (ini) at $0 \mathrm{~V}$ and the layer thickness $P: p_{0}=\left[\left(n_{p}\right.\right.$ (ini) $\left.\left./ n_{\max }\right) d-P\right] /(d-P)$. Note that $P$ for $V_{A}>V_{\text {thresh }}$ can be found with Eq. (11), and $P=0$ for $-V_{\text {thresh }}<V_{A}<V_{\text {thresh }}$ and $P$ for $V_{A}<-V_{\text {thresh }}$ can be calculated with Eq. (11) based on symmetry. The optical response is found by the approximated model, Eq. (9).
This results in steady-state gray levels above the threshold voltage:

$$
\begin{gathered}
R=e^{-\left(P / \delta_{\mathrm{ev}}\right)}-p_{0} e^{-\left(P / \delta_{\mathrm{ev}}\right)} \quad\left(V>V_{\mathrm{thresh}}=\frac{N_{+} e}{\varepsilon_{0} \varepsilon_{s}} d_{s}\right) \\
R=1-p_{0} \quad\left(V<V_{\text {thresh }}=\frac{N_{+} e}{\varepsilon_{0} \varepsilon_{s}} d_{s}\right) .
\end{gathered}
$$

Since $p_{0}$ is at most only a few percent, the optical response below the threshold, see Eq. (14), is close to the maximum reflectivity.

\section{B. Region I}

Now we investigate the dynamics in region I of Figs. 4(b) and 6(b). Here the starting situation is the steady state for a negative voltage $V_{P}<-V_{\text {thresh }}$ (bright state) which is the mirror situation of Fig. 8. At $t=0 \mathrm{~s}$, the voltage is switched to a voltage $V_{A}>V_{P}$ and the electric field in the bulk increases by approximately $\left(V_{A}-V_{P}\right) / d$ (Fig. 7). As a result, the particles in the bulk move to the left interface and their concentration near the left interface is approximately proportional with time and with $\left(V_{A}-V_{P}\right) / d$. This explains the linear decrease (proportional with $V_{A}-V_{P}$ ) in reflectivity in Fig. 6 (right) and in Fig. 4(d). Note that this indicates that there are many positively charged particles in the bulk and that the field in the bulk in steady state is zero (otherwise it would take much more time before particles could reach the left interface). The simulation indicates that the accumulated particles are not forming a compact layer close to the surface. This is because negative countercharges are released from the left interface and keep the field close to zero. Depending on the actually applied voltage $\left(V_{A}\right)$, the number of particles 
accumulating at the optical interface will either be reduced again (region II) or will increase again (region III).

\section{Region II}

When the applied voltage $V_{A}$ has a negative amplitude, we know that the positive particles cannot remain in a double layer near the left interface in steady state. The simulation indicates that the positive particles near the left interface (attract and) promote the release of negative countercharges adsorbed at that interface. Then these charges diffuse together away from the left interface. The time-dependent reflectivity shows a dip in reflectivity around the screening time $t=20 \mathrm{~ms}$.

\section{Region III}

Region III is characterized by a switch to a gray or black state after the screening time. In particular, for $V_{A}=4 \mathrm{~V}$ in Fig. 4(b), it can be noticed that first the reflectivity increases until after a delay (10-50 ms) when a sudden switch occurs to a gray or dark steady-state value. This observation indicates that two different mechanisms are at work simultaneously. The first mechanism is similar to that described for region II. The second mechanism is triggered by the depletion of the reservoir of adsorbed negative charges at the left interface, resulting in a negative electric field near that electrode and the compaction of particles.

\section{E. Current measurements}

The current measurements of Fig. 3 when the voltage is switched from $0 \mathrm{~V}$ to $V_{A}$ are characterized by an

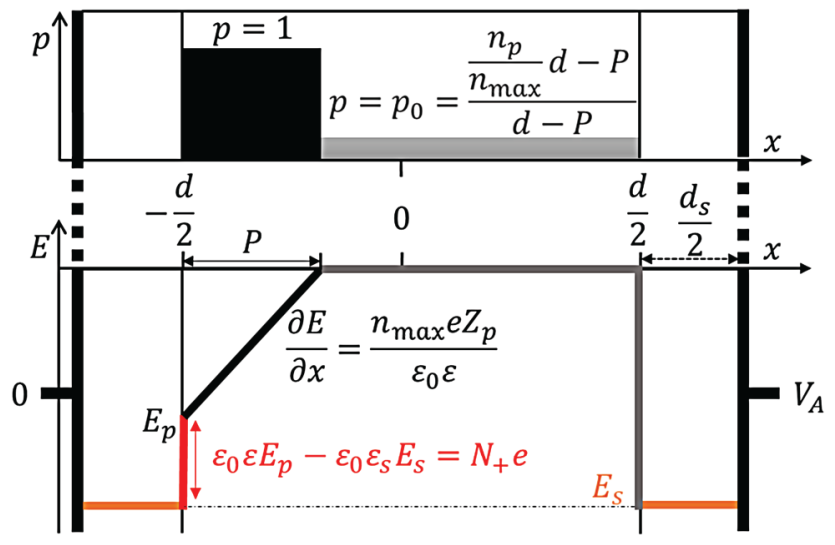

FIG. 8. Analytic model for the particle and field distribution in steady state for $V_{A}>V_{\text {thresh }}$. The positive surface charge results in a jump in the electric field at the left due to $N_{+} e$ and at the right due to adsorbed countercharges $N_{r-} e$ and fixed surface charges $N_{+} e$. Particles are compacted $(p=1)$ at the left side giving rise to a linear increase in the electric field. The charge of the noncompacted particles $\left(p=p_{0}\right)$ in the bulk is compensated by countercharges (not shown) giving a constant and zero electric field. initial exponential decay. The exponential decay corresponds to the charging of a capacitor $C$ (the two dielectric coatings) in series with a resistor $R$ (the liquid) with time constant $\tau=R C$. We found that for the bulk conductivity $\sigma=\left(I_{0} d / V_{A} S\right)=135 \mathrm{nS} / \mathrm{m}$ and resistance $R=$ $\left(V_{A} / I_{0}\right)=3 \times 10^{5} \Omega$. Using the theoretical value for $C=$ $\left(\varepsilon_{s} \varepsilon_{0} S / d_{s}\right) \quad\left(\varepsilon_{s}=3, S=6.25 \mathrm{~cm}^{2}\right)$, we find decay constants of 50 and $12 \mathrm{~ms}$ for coating thicknesses, respectively, of 50 and $200 \mathrm{~nm}$, corresponding well to the measured values (47 and $11 \mathrm{~ms}$ ).

The former approximation is valid as long as only a fraction of all bulk charges needs to be displaced toward the interfaces in order to achieve screening. This is satisfied if the charge required for screening $Q_{\text {scr }}$ is much smaller than the bulk charge $Q_{\text {bulk: }}$ :

$$
Q_{\text {scr }}=C V=\frac{\varepsilon_{0} \varepsilon_{s} S}{d_{s}} V \ll Q_{\text {bulk }} .
$$

An estimation for the total bulk charge $Q_{\text {bulk }}$ is given by

$$
Q_{\text {bulk }}=n_{p} Z_{p} e S d+n_{-} e S d,
$$

and results with the given parameters in $Q_{\text {bulk }} \cong 2 \mu \mathrm{C}$ (in the measurements of Fig. 3, $Q_{\text {scr }}$ is at least a factor of 5 smaller than $Q_{\text {bulk }}$ ). For voltages higher than $10 \mathrm{~V}$ and coating thicknesses smaller than $50 \mathrm{~nm}$, full charge separation can occur, and a different model is required such as that developed by Verschueren et al. [26].

The optical response in Fig. 4(a) starts with a normalized reflectivity of 0.93 , corresponding to a high reflectivity at $V_{P}=-10 \mathrm{~V}$. A voltage $V_{\text {thresh }} \cong 3 \mathrm{~V}$ is separating regions II and III as visualized in Fig. 4(a). Steady-state gray levels are observed between 0.93 (bright) and 0.05 (dark) as visualized in Fig. 4(e). For a negative voltage jump $\left(V_{A}<V_{P}\right)$, visualized in Figs. 4(b) and 4(c), a smooth transition to an even brighter state is observed due to the drift of particles out of the optical zone, then the reflectivity reduces again because particles diffuse back into the zero field [Fig. 4(e)].

\section{DISCUSSION}

In this section, we discuss what we can conclude from the relatively good agreement between the experimental results and the simulation results of our model.

\section{A. Surface charge}

The good agreement between measurements and simulations provides strong evidence that there is a fixed, positive surface charge present at the interfaces, which may be compensated by negative charges that may be adsorbed (but can be released) at that interface. On the other hand, the precise nature of these charges still remains unclear. Surface charging is a common mechanism in nonpolar liquids with surfactants. For example, colloidal particles 
acquire electrical charge on their surfaces depending on the type and concentration of surfactant additives. It is still unsure if the surface charges of these colloidal particles are the result of the dissociation of a surface ion or the adhesion of a charged micelle, nor is it fully understood which factor determines the amount and polarization [27-29]. Tests of substrates covered with other dielectrics show different switching behaviors and different threshold voltages, suggesting that the surface material plays an important role. The positive charge at both interfaces is observed to be stable over a timescale of minutes, but may gradually change over the course of days. We have observed that the change is faster at elevated temperatures $\left(T=70{ }^{\circ} \mathrm{C}\right)$.

\section{B. Charges in the bulk}

Pure nonpolar solvents are unable to dissolve typical molecular ions due to their small size $(<1 \mathrm{~nm})$ compared to the large Bjerrum length of approximately $28 \mathrm{~nm}$. When a surfactant is added, inverse micelles, which are much larger, may form. Based on their size compared to the Bjerrum length, a fraction of these inverse micelles will be charged [22]. In our simulations, we assume a fixed number of negatively charged inverse micelles with charge $+e$ and diameter $7.2 \mathrm{~nm}$ that are subject to drift and diffusion and may be adsorbed and released from the interfaces. Uncharged inverse micelles are not taken into account in our model. Note that a good agreement between measurements and simulations is obtained by assuming that there are no mobile positive inverse micelles. Such a lack of positive micelles in the device may be related to the large amount of positive charge that is present on the particles and on the interfaces of the dielectric coatings and/or to the probability of recombination with the large concentration of negative inverse micelles. However, simulation results suggest that low concentrations of positive micelles could be present in some cases. If a fraction of the total charge up to a few percent is in the form of positively charged inverse micelles, the simulation results in the case of blocking boundary conditions still fit well with the measurements. However, if the amount of charge represented in positive micelles is above a few percent of the total charge, the simulation results significantly deviate from the measurements since simulated gray levels start to fade away in steady state due to screening of the electric field by these positive micelles. In the case of an adsorption and release boundary condition, even with very small concentrations of positively charged micelles, the simulated gray levels start to fade away. Based on these observations and for simplicity, positive micelles are ignored in the proposed model.

\section{Boundary condition for countercharges}

Two kinds of boundary conditions for the negative inverse micelles are compared by numerical simulation: the boundary condition of adsorption and release [12] (where the inverse micelles stick to the surface when they touch it and are released when the field is zero) and the blocking boundary condition [30] (where the inverse micelles form a diffuse double layer or Debye layer with thickness $\lambda_{\mathrm{DL}}=\sqrt{\left(\varepsilon \varepsilon_{0} k_{B} T / 2 q^{2} n_{-}\right)} \approx 100 \mathrm{~nm}$ near the interface when the electric field is directed from the liquid to the solid). There is a subtle but important difference in the location of the negative charges in steady state between the two kinds of boundary conditions. In the case of an adsorbed layer of negative charge, the electric field in the bulk remains zero up to the solid-liquid interface. In the case of a diffuse double layer, the electric field in the double layer is pointing toward the interface and this keeps the positively charged pigment particles away from the interface. Because the Debye length is similar in length to the penetration depth of the evanescent field, we would in this case expect a nearly white reflectance. This does not correspond to the observations, and therefore, we prefer the adsorption/release boundary condition.

\section{Approximations}

In our model, we have neglected leakage current through the dielectric layers and micelle generation. In measurements, we see a non-negligible steady-state current though it is at least 100 times lower than the initial current. This current may be related to the observed slow drift of the threshold voltage.

Our optical model for reduction of the reflectivity does not take into account the refractive index of the particles or scattering. Nevertheless we think the present model describes the most important mechanism of absorption.

In the simulation, the particles are modeled as point charges with a maximal concentration instead of particles with a volume and charge distributed over it. Improving this model might lead to small corrections, but we think that the current model is able to explain the most important features in the measurements.

\section{CONCLUSION}

In this paper, we present dynamic electrical and optical measurements for an electrophoretic ink between two parallel electrodes coated with the poly(p-xylylene) polymer Parylene C, observed under TIR. The electrical measurements show that the device behaves as an RC circuit at low voltages, which can be understood from the conductive bulk liquid that is in series with the capacitive coating. The amount of charge inside the cell is sufficient to screen the electric field in the bulk of the liquid in steady state. The optical measurements provide insight into the particle concentration near the reflecting interface. Different regimes are observed in the dynamics of 
reflectivity when switching from one voltage to another, and in steady state a threshold voltage for gray levels is observed.

Based on the observations, an electro-optical model is presented based on positively charged pigment particles, negative countercharges, and positive surface charge fixed at the solid-liquid interface. The combined effect of drift and diffusion, adsorption and release of charges at the interfaces, and the effect of screening of the electric field can then explain the observed switching and the threshold voltage in the steady-state gray levels. We give an analytic solution for the steady-state gray levels and a physical explanation of the regimes based on the concentration of countercharges and particles at the surface. Screening of the bulk field caused by the dielectric coating is an important feature to realize switching without delay and a fast stabilization of steady-state gray levels.

Whereas previous research has often focused on low particle concentrations, this investigation is based on ink mixtures with high particle concentrations, which are very relevant for actual display applications. The different models that have been developed to explain the transient and steady-state electro-optical behavior are instrumental to understand and further improve electrophoretic ink displays.

\section{ACKNOWLEDGMENTS}

The authors would like to acknowledge Merck KGaA for providing the ink and the Research FoundationFlanders (FWO) for funding through a Strategic Basic Research grant.

[1] P. F. Bai, R. A. Hayes, M. Jin, L. Shui, Z. C. Yi, L. Wang, X. Zhang, and G. Zhou, Review of paper-like display technologies (Invited Review), Prog. Electromagn. Res. 147, 95 (2014).

[2] H. Liu, Y. Yao, Y. Wang, and W. Wu, Full-color reflective display system based on high contrast gratings, J. Vac. Sci. Technol. B 32, 06FE04 (2014).

[3] E. Herz, Electrophoretic Display Technology: The beginnings, the improvements, and a future in flexible electronics (2006).

[4] M. A. Mossman, L. A. Whitehead, and S. P. Rao, P-83: Grey scale control of TIR using electrophoresis of suboptical pigment particles, SID Symp. Dig. Tech. Pap. 33, 522 (2002).

[5] L. A. Whitehead and M. A. Mossman, Wide viewing angle reflective display, US 2004/0174584A1 (2005).

[6] Y. Chen, J. Au, P. Kazlas, A. Ritenour, H. Gates, and M. McCreary, Electronic paper: Flexible active-matrix electronic ink display, Nature 423, 136 (2003).

[7] C. B. Park, K. M. Kim, J. E. Lee, H. Na, S. S. Yoo, and M. S. Yang, Flexible electrophoretic display driven by solution-processed organic TFT with highly stable bending feature, Org. Electron 15, 3538 (2014).

[8] M. E. Parent, J. Yang, Y. Jeon, M. F. Toney, Z. L. Zhou, and D. Henze, Influence of surfactant structure on reverse micelle size and charge for nonpolar electrophoretic inks, Langmuir 27, 11845 (2011).

[9] F. Strubbe, F. Beunis, M. Marescaux, B. Verboven, and K. Neyts, Electrokinetics of colloidal particles in nonpolar media containing charged inverse micelles, Appl. Phys. Lett. 93, 11 (2008).

[10] F. Beunis, F. Strubbe, M. Karvar, O. Drobchak, T. Brans, and K. Neyts, Inverse micelles as charge carriers in nonpolar liquids: Characterization with current measurements, Curr. Opin. Colloid Interface Sci. 18, 129 (2013).

[11] F. Strubbe, M. Prasad, and F. Beunis, Characterizing generated charged inverse micelles with transient current measurements, J. Phys. Chem. A 119, 1957 (2015).

[12] M. Prasad, F. Strubbe, F. Beunis, and K. Neyts, Space charge limited release of charged inverse micelles in non-polar liquids, Phys. Chem. Chem. Phys. 18, 19289 (2016).

[13] K. Neyts, M. Karvar, O. Drobchak, T. Brans, F. Strubbe, and F. Beunis, Simulation of charge transport and steady state in non-polar media between planar electrodes with insulating layers, Colloids Surf. A Physicochem. Eng. Asp. 440, 101 (2014).

[14] K. Neyts, F. Beunis, F. Strubbe, M. Marescaux, B. Verboven, M. Karvar, and A. Verschueren, Charge transport and current in non-polar liquids, J. Phys. Condens. Matter 22, 494108 (2010).

[15] G. S. Roberts, R. Sanchez, R. Kemp, T. Wood, and P. Bartlett, Electrostatic charging of nonpolar colloids by reverse micelles, Langmuir 24, 6530 (2008).

[16] S. K. Sainis, V. Germain, C. O. Mejean, and E. R. Dufresne, Electrostatic interactions of colloidal particles in nonpolar solvents: Role of surface chemistry and charge control agents, Langmuir 24, 1160 (2008).

[17] F. Beunis, F. Strubbe, K. Neyts, and D. Petrov, Beyond Millikan: The Dynamics of Charging Events on Individual Colloidal Particles, Phys. Rev. Lett. 108, 016101 (2012).

[18] F. Strubbe, F. Beunis, T. Brans, M. Karvar, W. Woestenborghs, and K. Neyts, Electrophoretic Retardation of Colloidal Particles in Nonpolar Liquids, Phys. Rev. X 3, 021001 (2013).

[19] T. Lin, T. E. Kodger, and D. A. Weitz, Transport of charged colloids in a nonpolar solvent, Soft Matter 9, 5173 (2013).

[20] K. H. Lenssen, P. J. Baesjou, F. P. M. Budzelaar, M. H. W. M. Van Delden, S. J. Roosendaal, L. W. G. Stofmeel, A. R. M. Verschueren, J. J. Van Glabbeek, J. T. M. Osenga, and R. M. Schuurbiers, Novel Design for Full-Color Electronic Paper, SID, 685 (2008).

[21] M. S. Kilic, M. Z. Bazant, and A. Ajdari, Steric effects in the dynamics of electrolytes at large applied voltages. II. Modified Poisson-Nernst-Planck equations, Phys. Rev. E 75, 021503 (2007).

[22] F. Strubbe and K. Neyts, Charge transport by inverse micelles in non-polar media, J. Phys. Condens. Matter 29, 453003 (2017). 
[23] A. Rose, Space-charge-limited currents, Phys. Rev. 97, 1538 (1955).

[24] F. Beunis, F. Strubbe, M. Marescaux, J. Beeckman, K. Neyts, and A. R. M. Verschueren, Dynamics of charge transport in planar devices, Phys. Rev. E 78, 011502 (2008).

[25] S. Torquato, T. M. Truskett, and P. G. Debenedetti, Is Random Close Packing Well Defined?, Phys. Rev. Lett 84, 2064 (2000).

[26] A. R. M. Verschueren, P. H. L. Notten, L. J. M. Schlangen, F. Strubbe, F. Beunis, and K. Neyts, Screening and separation of charges in microscale devices: complete planar solution of the Poisson-Boltzmann equation, J. Phys. Chem. B 112, 13038 (2008).
[27] C. Calero, J. Faraudo, and D. Bastos-González, Interaction of monovalent ions with hydrophobic and hydrophilic colloids: Charge inversion and ionic specificity, J. Am. Chem. Soc. 133, 15025 (2011).

[28] M. M. Gacek and J. C. Berg, The role of acid-base effects on particle charging in apolar media, Adv. Colloid Interface Sci. 220, 108 (2015).

[29] J. Lee, Z. L. Zhou, G. Alas, and S. H. Behrens, Mechanisms of particle charging by surfactants in nonpolar dispersions, Langmuir 31, 11989 (2015).

[30] M. Karvar, F. Strubbe, F. Beunis, R. Kemp, N. Smith, M. Goulding, and K. Neyts, Charging dynamics of aerosol OT inverse micelles, Langmuir 31, 10939 (2015). 